\title{
Propuesta para estudiar agroecología con estudiantes de segundo ciclo del circuito escolar $N^{\circ} 4$ del cantón de Alvarado de Cartago
}

Proposal to study agroecology with students of second cycle of the educational circuit $\mathrm{N}^{\circ} 4$ of the canton of Alvarado in Cartago

\section{Volumen 20, Número 1}

Enero - Abril

pp. 1-28

\section{Elena Fernández Monge}

\section{Citar este documento según modelo APA}

Fernández Monge, Elena. (2020). Propuesta para estudiar agroecología con estudiantes de segundo ciclo del circuito escolar $\mathrm{N}^{\circ} 4$ del cantón de Alvarado de Cartago. Revista Actualidades Investigativas en Educación, 20(1), 1-28. Doi. 10.15517/aie.v20i1.40178 


\title{
Propuesta para estudiar agroecología con estudiantes de segundo ciclo del circuito escolar $\mathrm{N}^{\circ} \mathbf{4}$ del cantón de Alvarado de Cartago
} Proposal to study agroecology with students of second cycle of the educational circuit $\mathrm{N}^{\circ} 4$ of the canton of Alvarado in Cartago

\section{Elena Fernández Monge ${ }^{1}$}

\begin{abstract}
Resumen: La presente investigación fue construida por la inminente necesidad de insertar la agroecología en el currículum educativo desde un enfoque dinámico e integrador, en un contexto donde la agricultura basada en el uso intensivo de agroquímicos representa la principal actividad productiva y económica de la zona que comprende el cantón de Alvarado. Metodológicamente, se realizó una investigación cualitativa-descriptiva, se eligieron tres centros educativos del cantón del circuito escolar №4 y de estos participaron un grupo de estudiantes y docentes de segundo ciclo. Se utilizaron como instrumentos y técnicas de recolección de información la búsqueda bibliográfica, la observación sistemática, las entrevistas individuales, los grupos focales con docentes, así como tres técnicas grupales y una individual con estudiantes. Además, se conoció una experiencia pedagógica presencial dirigida a formadores, basada en metodologías alternativas de enseñanzaaprendizaje para estudiar los sistemas agroalimentarios locales. Los resultados muestran que actualmente no existe una formación exclusiva, suficiente y pertinente orientada en el estudio de los sistemas agroalimentarios locales. Resulta apremiante la creación de espacios de acción, reflexión y sensibilización respecto a la agroecología, para los cuales las técnicas o métodos de enseñanza-aprendizaje empleados son determinantes. El producto elaborado consiste en una guía didáctica dirigida al personal docente para abordar la agroecología desde las materias de Ciencias, de Estudios Sociales y de Cívica.
\end{abstract}

Palabras clave: agroecología, educación ambiental, salud ambiental, educación primaria.

Abstract: The present research was build given the imminent necessity of including agroecology in the educational curricula from a dynamic and integrative approach, in a context where agriculture based on the intensive use of agrochemicals is the main productive and economic activity at Alvarado municipality. Methodologically, the research was qualitative-descriptive: three schools were chosen to represent the education circuit Number 4, and a group of students and teachers of the higher (second) cycle participated. Bibliographic research, observations, individual interviews and focal groups with teachers and students were used as tools for collecting the information. Moreover, a teaching experience based in alternative methods was used to study the local agri-food systems. The results show that currently there is no an specific and sufficient training oriented to the knowledge of local agrifood systems. Thus, it is important to create spaces for the action, reflection, and sensitization towards agroecology, for which teaching-learning methods and technics are determinant. The final product is an educational guide on agroecology for teachers, to be used in Science, Social Study and Civics.

Keywords: agroecology, environmental education, environmental health, primary education.

\footnotetext{
1 Ministerio de Salud, Costa Rica. Licenciada en Salud Ambiental de la Universidad de Costa Rica. ORCID https://orcid.org/0000-0002-2252-3983
}

Dirección electrónica: laelefernandez@gmail.com

Artíuclo recibido: $1^{\circ}$ de julio, 2019

Enviado a corrección: 11 de setiembre, 2019

Aprobado: 18 de noviembre, 2019 


\section{Introducción}

La provincia de Cartago, en Costa Rica, es una de las regiones de mayor producción agrícola en el país. Dicha actividad genera la mayoría de empleos y se caracteriza por el uso intensivo de agroquímicos. De acuerdo con De la Cruz et al. (2004), esta situación provoca, a su vez, un riesgo latente para la salud de las personas y los recursos naturales, ya que una vez liberados al ambiente, muchos de sus componentes pueden persistir durante años y se pueden dispersar por muchos kilómetros desde el lugar en el que fueron aplicados. Aunado a esto, por su composición química, estos insumos sintéticos tienen distintos grados de toxicidad, lo que puede provocar efectos adversos en la salud humana (MSAL-SAyDS-OPSAAMMA, 2007).

La población infantil es más susceptible que la de las personas adultas a los efectos por exposición a agroquímicos, y el riesgo es mayor si los sistemas de cultivos agrícolas forman parte del entorno diario en donde los niños y las niñas crecen. La mayoría de estos problemas de salud ambiental ocurren en zonas rurales, en viviendas que se ubican cerca de grandes extensiones de monocultivos y en hogares que poseen parcelas donde la niñez se involucra en el trabajo y entra en contacto con estas sustancias (Programa de las Naciones Unidas para el Medio Ambiente [PNUMA]; 2004).

Es por ello que resulta prioritario llevar a cabo estrategias de prevención de conductas y acciones de riesgo, con un abordaje integral, en las que el sector educativo desempeñe una función muy importante, ya que muchas veces las desfavorables consecuencias de este problema en la salud humana y el ambiente van de la mano con la desinformación de las personas, quienes carecen de acceso a espacios de información y aprendizaje oportunos. Actualmente, en Costa Rica, el abordaje de la agricultura en el sector escolar representa un vacío, pues desde el año 2013, como resultado de un acuerdo emitido por el Consejo Superior de Educación, se eliminó la asignatura de Educación Agrícola del plan de estudios de I y II Ciclo de la Educación General Básica, y se incorporaron sus contenidos a la asignatura de Ciencias (Carvajal, 2013). Por lo tanto, el tema se aborda dentro de otras materias, pero quizás no con la atención y el detalle que requiere.

Estas razones fundamentan la necesidad de elaborar una propuesta de enseñanzaaprendizaje y concienciación, enfocada en promover la agroecología en la población escolar como una alternativa al modelo de producción agrícola actual que prevalece en la zona geográfica de estudio. 
En este abordaje, la Salud Ambiental, en general, y la Educación Ambiental, en particular, se constituyen en áreas del conocimiento centrales para plantear y desarrollar este tipo de acciones. Al estudiantado se le debe presentar la relación entre las acciones cotidianas, la salud y el ambiente que les rodea, en aras de generar conocimientos para la prevención de enfermedades ambientales actuales, y así promover un cambio desde la base del sistema y lograr instaurar hábitos duraderos y transmisibles que repercutan en una mejor salud y calidad de vida (Fundación Vivo Sano, 2018). Estos procesos deben introducirse a través de la Educación Ambiental desde la niñez, y por ende, la escuela constituye un elemento imprescindible en esta dirección.

El objetivo de esta investigación fue Diseñar una propuesta educativa dirigida al profesorado del segundo ciclo del circuito escolar $\mathrm{N}^{\circ} 4$ del cantón de Alvarado de Cartago, para promover la agroecología con la población estudiantil.

Dicha propuesta consistió en la elaboración de una guía didáctica para incluir la agroecología dentro de los planes de estudio de las materias de Ciencias y Estudios Sociales-Cívica. Fueron posibles gracias a la participación de estudiantes y docentes del segundo ciclo de primaria de tres escuelas de dicho cantón, tomando como base el contexto agrícola de la zona.

Además para cumplir con los objetivos específicos propuestos se realizaron las siguientes acciones:

- Caracterizar la actividad agrícola del cantón de Alvarado.

A través de este objetivo fue posible obtener información precisa relacionada con la caracterización geográfica, geológica, hidrográfica y climática de la zona en estudio, así como su relación con la agricultura, la cual es una actividad predominante y forma parte del entorno diario de sus habitantes.

- Explorar la percepción del estudiantado con respecto a la agricultura de la zona.

Este acercamiento ha permitido conocer el grado de conocimiento que tienen sobre el tema, y el aprendizaje que han obtenido a través del sistema educativo, contribuyendo así con la construcción de la propuesta.

- Describir la forma en que el personal docente incorpora el tema de la agricultura dentro del programa de estudios.

De esta manera se identificó el modo en que actualmente se estudia la agricultura y las oportunidades que ofrecen los planes de estudios de Ciencias y Estudios Sociales-Cívica. Aunado a esto, se indagó sobre la manera en que las personas docentes estudiarían la 
agroecología, partiendo de sus expectativas, sugerencias, inquietudes y posibles recursos de los que disponen.

- Analizar una experiencia pedagógica dirigida a formadores basada en metodologías e iniciativas alternativas de enseñanza-aprendizaje para estudiar los sistemas agrícolas locales basados en la agroecología con estudiantes.

Para ello fue posible conocer de manera presencial la fase intensiva del Diplomado "Alimentación, Comunidad y Aprendizaje" del programa "Laboratorios para la Vida", el cual es un programa de formación dirigido a docentes y tiene lugar cada año en San Cristóbal de las Casas, Chiapas, México. La finalidad ha sido indagar la forma en que docentes y formadores de otras latitudes abordan la agroecología y promueven los huertos escolares con estudiantes de primaria. Esto ha contribuido en la adquisición de algunas herramientas para el proceso de enseñanza-aprendizaje de la agroecología, las cuales han servido de insumo para incluir en la propuesta final.

\section{Referente teórico}

La Ley de desarrollo, promoción y fomento de la actividad agropecuaria orgánica, № 8542 define la actividad agropecuaria como: "Actividad agropecuaria basada en la homogenización de los sistemas de producción, el aislamiento del producto de los elementos del ambiente, la labranza mecánica, la nutrición y la protección artificial, utilizando agroquímicos sintéticos y energía fósil" (2006, Capítulo II, Artículo 5, inciso b.).

Sin embargo, es sabido que los productos agroquímicos generan fuertes impactos ambientales, y es que una vez liberados, muchos de sus componentes pueden persistir durante años y se pueden dispersar miles de kilómetros desde el lugar en el que fueron aplicados causando grandes daños a la naturaleza y afectando la salud de todos los seres vivos, organismos acuáticos y terrestres, insectos polinizadores, aves y demás fauna silvestre (De la Cruz et al., 2004).

Las sustancias agroquímicas pueden provocar efectos adversos si penetran en el cuerpo, ya que por su composición química estos insumos tienen distintos grados de toxicidad. Los más tóxicos son particularmente peligrosos, inclusive en pequeñas cantidades, y el peligro se acrecienta al introducir estos productos masivamente al ambiente, como consecuencia de una inadecuada aplicación, así como por la acumulación e incorrecta disposición final de los envases. Estos se desplazan por todo el ambiente hasta llegar 
directamente a las personas a través del agua, el aire, el suelo, e indirectamente a través de los alimentos (MSAL-SAyDS-OPS-AAMMA]; 2007).

Algunos de los principales efectos crónicos en la salud asociados con el uso de agroquímicos son el cáncer, trastornos del sistema nervioso, problemas reproductivos, efectos en el sistema inmunológico y alteración del sistema endocrino. En el caso del sistema nervioso, puede traducirse en inteligencia disminuida, trastornos en la conducta y efectos en el desarrollo del cerebro en su etapa prenatal. Los problemas reproductivos se relacionan con defectos congénitos, infertilidad, nacimientos prematuros y abortos, y en cuanto al sistema endocrino, pueden imitar o bloquear las hormonas o provocar una actividad hormonal inadecuada, entre otros problemas (Weinberg, 2009).

La población infantil es la más vulnerable a los efectos de los agroquímicos por varios factores de riesgo. La mayor vulnerabilidad de este sector poblacional se debe a razones de carácter fisiológico, entre ellos, el rápido crecimiento, el desarrollo de los órganos y el metabolismo diferente, lo que hace que descompongan, excreten y desactiven dichas sustancias de manera distinta. El riesgo se acrecienta si viven cerca de plantaciones o si ayudan en el trabajo familiar, donde hacen uso de agroquímicos. Aunado a esto, por su inexperiencia, falta de maduración, analfabetismo e incapacidad para evaluar los peligros, esta población se vuelve más vulnerable, algo que podría prevenirse si existiera mayor alerta ante el riesgo (PNUMA, 2004).

Es decir, el uso de agroquímicos sintéticos supone un riesgo para la Salud Ambiental, la cual es definida por la OMS (1993) como:

Todos aquellos aspectos de la salud humana, incluyendo la calidad de vida, que están determinados por factores ambientales, físicos, químicos, biológicos, sociales y psicológicos en el ambiente. También se refiere a la teoría y práctica de valorar, corregir, controlar y evitar aquellos factores en el ambiente que potencialmente puedan perjudicar la salud de generaciones actuales y futuras. (Gosselin, Furgal y Ruiz, 2001, p.3)

En este contexto se destaca, entonces, el concepto de Educación Ambiental, que es de vital importancia para generar un pensamiento crítico sobre el ambiente, sustentado en acciones, información y prevención de daños a partir de la educación en la escuela primaria. En este nivel educativo es fundamental crear este tipo de pensamiento enfocado en la capacidad de identificar y solucionar problemas ambientales (UNESCO et al., 1997). Para 
ello es importante crear planes de estudio de tipo transversal en la Educación Ambiental. Este enfoque es definido por la Comisión Nacional Ampliada de Transversalidad (2002) de la siguiente manera:

Enfoque pedagógico que aprovecha las oportunidades que ofrece el currículo, incorporando en los procesos de diseño, desarrollo, evaluación y administración curricular, determinados aprendizajes para la vida, integradores y significativos, dirigidos al mejoramiento de la calidad de vida individual y social. Es de carácter holístico, axiológico, interdisciplinario y contextualizado. (Ministerio de Educación Pública [MEP], 2004, p. 2)

En esta línea, y ante la necesidad de un plan curricular de Educación Ambiental es importante tomar en cuenta los siguientes conceptos para la incorporación de contenidos, prácticas y experiencias en los planes de estudio escolares:

Actividad agropecuaria orgánica: Toda actividad agropecuaria y su agroindustria que se sustenta en sistemas naturales para mantener y recuperar la fertilidad de los suelos, la diversidad biológica y el manejo adecuado del recurso hídrico, y que propicia los ciclos biológicos en el uso del suelo. Desecha el uso de agroquímicos sintéticos, así como el uso de organismos transgénicos. Además, contribuye al equilibrio ambiental, tiende a un equilibrio sociocultural de las formas de organización comunitaria indígena y campesina, integra a las prácticas actuales los conocimientos tradicionales, genera condiciones laborales justas, defiende el derecho de las personas a producir alimentos sanos y prioriza el uso de recursos locales (Asamblea Legislativa de la República de Costa Rica, 2007)

Agroecología: Se puede entender como un enfoque de la agricultura, cuyos principios están ligados al ambiente y a la sociedad. Los procesos que conlleva están centrados no solamente en un modelo de producción, sino en la sostenibilidad ecológica de este, por lo que el área de cultivo es la imitación de un ecosistema en donde se dan interacciones ecológicas y una serie de procesos ecológicos (Restrepo, Angel, Praguer, 2000). Con base en el conocimiento de estas relaciones y procesos, se busca la manera en que los sistemas agrícolas dependan menos del uso de insumos externos y se manejen mediante prácticas sostenibles que minimicen su impacto al ambiente y a la salud humana (Restrepo et al., 2000).

Según la Organización de las Naciones Unidas para la Agricultura y la Alimentación [FAO] (2010), los huertos agroecológicos se perfilan como una alternativa a nivel local para 
atender la problemática y las necesidades urgentes que enfrenta la humanidad, tales como el cambio climático, en especial, en países cuyas economías dependen de la actividad agrícola. También refiere que podrían responder a otra serie de necesidades que deben abordarse, tales como la seguridad alimentaria básica, que se encuentra amenazada; el encarecimiento de los insumos, así como la pérdida de conocimientos agrícolas.

Soberanía alimentaria: Se trata de un concepto desarrollado por Vía Campesina en la Cumbre Mundial sobre la Alimentación en 1996. Es un movimiento global que se compone por millones de campesinos y campesinas, pequeños y medianos productores, y otros sectores sociales como pueblos desterrados, indígenas, migrantes, grupos medioambientales, grupos de consumidores, asociaciones de mujeres, pescadores, y otros trabajadores. Se opone firmemente a las políticas neoliberales, a los agronegocios y a las multinacionales que están destruyendo los pueblos y la naturaleza. Actualmente, cuenta con el reconocimiento de numerosas instituciones y gobiernos (La Vía Campesina, 2003).

\section{Metodología}

\subsection{Enfoque}

La investigación empleó el método cualitativo-descriptivo. De acuerdo con Danhke (1989), "los estudios descriptivos buscan especificar las propiedades importantes de personas, grupos, comunidades o cualquier otro fenómeno que sea sometido a análisis". De esta manera, se pretende recolectar y evaluar datos indispensables que permitan analizar la situación en estudio (Hernández, Fernández y Baptista, 2003). Según Taylor y Bogdan (1996, citados por Abarca, Rojas y Sibaja, 2013), dicho enfoque se refiere a “...la investigación que produce datos descriptivos: las propias palabras de las personas, habladas o escritas y la conducta observable". En este sentido, para alcanzar la propuesta final fue indispensable el aporte de las personas participantes desde su experiencia de vida, sus percepciones, conocimientos y opiniones. Parte de la información se obtuvo a través de fuentes primarias, en este caso, puntos de vista, actitudes y opiniones de los participantes. Por otro lado, la investigación también hizo uso de fuentes documentales, ya que se realizó una revisión bibliográfica para desarrollar los objetivos. 


\subsection{Población en estudio}

La población estuvo constituida por docentes y estudiantes del segundo ciclo de tres centros educativos del cantón de Alvarado ${ }^{2}$. Se eligieron en representación del circuito $\mathrm{N}^{\circ} 4$, de la Dirección Regional de Cartago con la finalidad de que la propuesta pueda ser aplicable a cualquier escuela del circuito. Las escuelas seleccionadas fueron: Escuela Guillermo Rodríguez Aguilar de Santa Teresa, distrito de Capellades; Escuela Encarnación Gamboa Piedra, distrito de Capellades y la Escuela Presbítero Juan de Dios Trejos, distrito de Pacayas. Los directores de estos centros se mostraron muy interesados y anuentes en colaborar y extendieron el permiso correspondiente.

Para la selección de los tres centros educativos se tomó en cuenta su tamaño y ubicación; los dos primeros son escuelas pequeñas ubicadas en el distrito de Capellades, mientras que el tercero posee la mayor población estudiantil y se ubica en el distrito de Pacayas, cabecera del cantón.

Las personas participantes fueron docentes y estudiantes de segundo ciclo. Se eligieron 62 alumnos de los niveles de cuarto, quinto y sexto grado (edades entre los 10 y 13 años), tanto hombres como mujeres, porque representan una población en edad ideal para asumir el compromiso de implementar acciones en promoción de la agroecología y por el nivel de conocimiento que pueden tener con respecto al tema en estudio de acuerdo con sus vivencias. Los estudiantes de sexto grado que participaron tuvieron oportunidad de recibir la materia de agricultura cuando estuvieron en el primer grado, a diferencia de los escolares de cuarto y quinto grado que participaron, quienes nunca la recibieron.

Los criterios para seleccionar a las y los estudiantes fue que estuvieran activos en el periodo escolar 2016, que vivieran en Pacayas y Capellades, por ser distritos del cantón en estudio, que estuvieran interesados en participar voluntariamente en las dinámicas y que contaran con el consentimiento informado de sus padres y madres.

Se eligieron en total 18 docentes que impartieran la materia de Ciencias y la de Estudios Sociales, ya que representan las asignaturas más pertinentes para el tema en estudio. Para la actividad grupal con docentes se abrió la posibilidad de que participaran todos los de las materias básicas de segundo ciclo que estuvieran interesados.

\footnotetext{
${ }^{2}$ El circuito $\mathrm{N}^{\circ} 4$ se localiza en la zona norte de la provincia de la provincia de Cartago y comprende un total de 25 escuelas públicas diurnas, distribuidas entre los cantones de Alvarado, Oreamuno y parte del cantón de Cartago (Tierra Blanca).
} 


\subsection{Técnicas de recolección}

Para el desarrollo del primer objetivo se utilizó la técnica de observación en varios puntos de la zona en estudio, definidos por la investigadora. Para ello se aplicó una guía validada previamente con el objetivo de obtener información sobre aspectos físicos del cantón de Alvarado, tales como las características geográficas, geológicas, hidrográficas, climáticas, de uso del suelo y de la actividad agrícola que se desarrolla. Para este último, también se realizaron conversaciones informales con agricultores de las comunidades de Capellades y Pacayas, cuyo aporte contribuyó en complementar la información sobre la caracterización de la actividad agrícola.

Para el segundo objetivo, se diseñaron y aplicaron cuatro técnicas grupales mediante dos talleres para cada escuela. Dos de ellas tuvieron trabajo en subgrupos. Una de las técnicas en subgrupos consistió en la caracterización de la comunidad mediante dibujos, y la otra fue sobre asociación de imágenes con frases, a través de la cual se indagó sobre el conocimiento que tienen con respecto a la agricultura convencional y la agroecología La tercera técnica fue una entrevista grupal, en la cual se compartieron vivencias y experiencias con respecto a la forma en que se abordó el tema de la agricultura dentro de la escuela, y además se indagó sobre los conocimientos, actitudes y opiniones que tienen con respecto a la agricultura de la zona. Por último, se realizó un trabajo individual con el fin de explorar sus inquietudes y expectativas en cuanto a la puesta en marcha de un huerto escolar.

Para el desarrollo del tercer objetivo, se emplearon dos técnicas: en primer lugar, entrevistas semiestructuradas a dos docentes por escuela, de las materias de Ciencias y Estudios Sociales. Y en segundo lugar, un grupo focal, con base en preguntas generadoras que se obtuvieron a partir de la información de las entrevistas. En total se realizó una sesión por escuela.

De acuerdo con Gurdián (2007), el grupo focal es una técnica cualitativa de recolección de información diseñada para aplicarse con grupos entre 6 y 12 personas, y consiste en una entrevista grupal que busca generar discusión de un tema especial vivido y compartido mediante experiencias en común. A través de esta técnica, las personas docentes aportaron comentarios y opiniones referentes a la inclusión de la agricultura dentro del actual programa de estudios del segundo ciclo, así como otra información sobre estrategias didácticas, evaluación y tiempo que invierten actualmente para la enseñanza de la agricultura. La información obtenida ha sido de gran utilidad para el diseño de la propuesta de esta investigación. 
Para la recolección de la información obtenida de docentes y estudiantes, se recurrió al uso de medios tecnológicos y didácticos, entre los que se mencionan la elaboración de mapas e ilustraciones por parte del grupo estudiantil, fotografías para generar una memoria de cada una las diferentes técnicas aplicadas con ambos grupos, y grabaciones de audio para las dos técnicas de entrevistas grupales aplicadas.

El desarrollo del cuarto objetivo fue posible a través de la observación participante. A partir de esta técnica, la investigadora logró conocer una serie de metodologías e iniciativas educativas utilizadas para el estudio de los sistemas agroalimentarios locales basados en la agroecología, y su abordaje con el estudiantado de primaria y secundaria de distintos centros educativos de esta región.

Importa recalcar que la revisión de los programas de estudio de segundo ciclo del Ministerio de Educación Pública de Ciencias (MEP, 2016) y de Estudios Sociales-Cívica (MEP, 2013) ha sido indispensable para la elaboración de la propuesta.

\subsection{Procesamiento y análisis}

Para al procesamiento y análisis se seleccionó un procedimiento inductivo- deductivo. La recopilación intensiva de información permitió aplicar la "triangulación metodológica", consistente en el uso de distintos instrumentos y técnicas de investigación para el estudio de un mismo objeto, con el fin de validar los datos obtenidos y con ello obtener resultados más satisfactorios y confiables (Colás y Buendía, 1998). En este caso, la triangulación se ha basado en la comparación de la información obtenida a través de los estudiantes, docentes, agricultores y la búsqueda bibliográfica. Asimismo, se utilizó la triangulación metodológica mediante la aplicación simultánea de diferentes técnicas e instrumentos ya mencionados para recopilar la información.

Posteriormente, se elaboró una base de datos en Excel para clasificar y transcribir manualmente la información obtenida a través de estos informantes, de la observación de campo, de la documentación consultada y de la experiencia presencial en el diplomado. Esto facilitó el procesamiento de la información a través de la técnica cualitativa de análisis de contenido. Cabe destacar que este análisis se hizo en función de las categorías y subcategorías de análisis planteadas para cada uno de los objetivos de la investigación.

Tanto las personas estudiantes como docentes participantes firmaron un consentimiento informado donde se les explicó el objetivo de la investigación, con el fin de que tomaran libre y voluntariamente la decisión de participar. Aunado a esto, se les garantizó 
la confidencialidad y el anonimato de los trabajos realizados y de las entrevistas. En relación con las consideraciones éticas, es necesario aclarar que la participación del estudiantado y del profesorado fue voluntaria, se respetó su derecho a la autonomía y se contó con el apoyo de los docentes y directores para darle oportunidad a los tres niveles de segundo ciclo.

\section{Resultados y discusión}

\subsection{Caracterización de la zona y de la actividad agrícola del cantón de} Alvarado

La caracterización ha sido importante para el objetivo principal de la investigación, ya que sirvió como insumo para elaborar las actividades de las guías, ajustándose a la realidad del contexto. La información recopilada mediante la búsqueda bibliográfica, la observación en trabajo de campo y las conversaciones con productores arrojó resultados muy similares entre sí, en cuanto al uso del suelo, el sistema productivo y su escala de producción, los cultivos predominantes y el tipo de prácticas, entre otros aspectos. El Plan Municipal de Gestión Integral de Residuos Sólidos para el Cantón de Alvarado (cuya última actualización fue en el 2014), indica que el cantón presenta 4 usos del suelo, que son: agricultura, ganadería, cobertura boscosa y zonas urbanizadas. Se enfatiza que el $74 \%$ corresponde a un uso agropecuario y un $24 \%$ a cobertura boscosa.

Este plan recalca que la producción agropecuaria constituye la principal actividad de Alvarado, lo cual se evidenció mediante el trabajo de campo, destacando el cultivo de hortalizas en el distrito de Pacayas, donde, además, se concentra el núcleo poblacional y en el centro de Capellades. Según datos de la Municipalidad de Alvarado (2015), la escala de producción predominante en Alvarado son las microempresas y las pequeñas empresas dedicadas a la agricultura, lo que revela que el cantón posee una economía de alta productividad en manos de pequeños productores. Aunado a esto, el sistema de producción de monocultivo basado en la aplicación constante de productos agroquímicos es lo más común. Es importante destacar que los procesos de post-cosecha de algunos cultivos, como por ejemplo los tubérculos, implican un gran consumo de recursos y contaminan el entorno natural.

De acuerdo con Naranjo (2010), la post-cosecha de la papa implica un lavado para remover la capa de tierra que lo cubre, con tal de obtener una presentación agradable en los mercados. Esta actividad comercial conocida como lavaderos, donde se lava papa y zanahoria, forma parte de la cadena de mercado de estos productos, la cual genera un gran 
impacto ambiental relacionado con un gran consumo de agua y la contaminación de los ecosistemas acuáticos por el inadecuado tratamiento de las aguas residuales.

Cabe destacar que el cultivo de la papa aporta el $45 \%$ de la producción a nivel nacional, es decir, es el cantón con mayor producción. En volumen, le siguen la zanahoria, el repollo, chile dulce, vainica y en las últimas décadas ha tomado fuerza el cultivo de cebolla (MAG, 2015).

En síntesis, lo anteriormente expuesto refleja que el modo de producir en el cantón de Alvarado se reduce al uso intensivo de agroquímicos, técnicas de monocultivo y otras prácticas cuyo fin es acelerar el proceso para lograr una mayor productividad. Sin embargo, estas prácticas están agotando los suelos, afectando el recurso hídrico y representan un riesgo para la salud de los seres vivos. Las escuelas del circuito en estudio comparten la característica de estar insertas en un ambiente agrícola de producción intensiva basada en el uso de sustancias sintéticas. En las visitas de campo no se logró conocer ninguna experiencia de personas que practiquen la producción agroecológica. Puede que existan productores inclinados hacia esta agricultura, pero en cualquier caso no es el tipo de producción predominante.

\subsection{Percepción del estudiantado con respecto a la agricultura de la zona}

Se logró recabar información importante sobre sus comunidades, el conocimiento que tienen del sistema agro-productivo que predomina en el cantón, la relación con el ambiente físico que les rodea, su percepción acerca de la agricultura ecológica y la agricultura basada en agroquímicos. Además, se logró discutir sobre la manera en que podrían diseñar un huerto escolar agroecológico.

Según lo observado, los y las estudiantes mostraron gran conocimiento sobre los recursos naturales y elementos del paisaje de la zona, las características geográficas y climatológicas, el uso del suelo, el tipo de cultivos que prevalecen y las prácticas y procedimientos que conllevan estas actividades desde la siembra hasta la cosecha, las cuales en muchos casos implican el uso de venenos. También demostraron una gran sensibilidad sobre los problemas que aquejan al ambiente, como los ríos contaminados con residuos y la deforestación. A continuación, se muestran algunos extractos de diálogos aportados por el estudiantado, tomados de las distintas actividades que se realizaron. Los y las estudiantes se identificaron como participante 1 hasta participante 62.

Con respecto a la influencia de las condiciones climáticas y su efecto en los cultivos: 
"En el caso de la zanahoria, yo sé que, si se siembra en lugares fríos, la zanahoria queda como de color pálido; si se siembra, un ejemplo aquí que el clima es caliente y frío, entonces queda de un color más anaranjado. $Y$ la siembra se inicia más que todo desde el verano y se termina en invierno, o en temporada de vacaciones, algo así. Depende del clima porque si es clima frío dura más" (Participante 52).

Con respecto a las prácticas de siembra:

“(...) bueno es que en mi casa un día sembraron, pero nada más vi, así como abren primero la tierra para sembrar culantro, sin barro, pero la tierra tiene que estar sin boronas. Entonces desmoronan la tierra, que queda sin piedras ni nada y le tiran la semilla, y luego le ponen eso y tienen que arrancarle el pasto que le crece alrededor" (Participante 48).

Con respecto al cultivo de frijol y el tomate, el grupo estudiantil describió algunos aspectos de su siembra y crecimiento, y según lo que parece, se utilizan técnicas que sus mayores han transmitido. Estas parecen ser prácticas que armonizan con el ambiente:

“(...) mi abuelito que yo le ayudo a recoger el frijol o a sembrar el frijol. Él agarra, un ejemplo, una bolsa de frijol tío pelón y lo pone a secar y después se le va naciendo la raíz de una parte, un extremo que tiene el frijol y después así se siembra. No se le echa ningún veneno" (Participante 61).

“Mi papá al tomate es que lo siembra y conforme va creciendo lo va amarrando (...). La mata que crece como a una altura de aquí y tiene que ir amarrando, porque si no se cae" (Participante 13).

Sobre cultivos que se germinan en bandejas o almácigos y luego son trasplantados:

“(...) un ejemplo en el zuchinni o en el zapallo, se mete la semilla que es una matica, no hay semilla, entonces se tiene que enterrar toda de tierra, hacer un círculo alrededor, sino la mata se muere" (Participante 61).

"(...) cuando se hace el zuquín o el zapallo, así en bandeja, hay veces que sale primero la raíz y en ese no es necesario que esté la tierra fina-fina, porque los terrones cuando va a salir los empuja para un lado y los levanta" (Participante 22). 
En cuanto al cultivo de la papa, algunos niños expusieron parte del proceso desde que se siembra hasta que se realiza la cosecha. La particularidad de este cultivo es que tal como lo explicaron los niños, la semilla es la papa misma

“(...) se alista la semilla y cuando ya le nace el rabito se alista el terreno y se va tirando la papa y se tapa. Uno lo alista en la casa o en un galerón. O si la papa no está muy buena la usan para semilla, la vuelven a utilizar" (Participante 15).

"(...) por ejemplo se hace la era y queda como un caño en el centro, se echa la papa ahí y después se tapa y nace la papa!" (Participante 58).

“(...) va creciendo, va creciendo y llega un punto en que va a tener una flor blanca, pero usted llega, bueno yo lo he visto así, que llegan y agarran eso, lo jalan así, y se hacen más papas, porque las papas se producen abajo de la tierra, entonces usted tiene que agarrar la mata (...)" (Participante 25).

Por otro lado, los y las estudiantes compartieron métodos en los cuales se utilizan sustancias venenosas o agroquímicos, sobre todo en lo que respecta al cultivo de la papa:

"Por ejemplo, mi papá nada más le echa solo dos tipos de veneno, una para el gusano y otro para que nazcan más rápido".

“(...) bueno lo único que sé de la papa que siembra mi papá, es que se tiene que sembrar en zonas frías y cuando ya la papa esté cultivada y ya esté lista para arrancarla, se le echa un veneno rosado, es como un polvo rosado, creo que es para que se mantenga" (Participante 55).

En síntesis, la concepción que tienen demuestra que la agricultura es muy importante en la zona y que las personas participantes conocen muy bien sobre su comunidad y las prácticas agrícolas que se dan. Cabe destacar que quienes tienen familiares cercanos que se dedican a la actividad fueron más participativos y conocen más al respecto.

Indistintamente de que los métodos que se utilicen sean aptos o no, existe mayor conocimiento sobre el tipo de cultivos y las técnicas de siembra o cosecha. Fue muy acertada la descripción que dieron sobre la caracterización de la agricultura de la zona, principalmente porque es muy homogénea y depende mucho de los ciclos del año. En cuanto al conocimiento que demuestran sobre la agroecología, los aciertos mostrados en su caracterización han sido más por intuición que por conocimiento. Queda muy claro que son estudiantes muy críticos y analíticos, y el grado escolar no define su nivel de conocimiento en 
el tema. Lo que realmente lo hace es la relación que tienen con la agricultura en su cotidianeidad (el entorno, las actividades y las personas que les rodean) y no tanto lo que se estudia en la escuela.

\subsection{Incorporación del tema de la agricultura dentro del programa de estudios según personal docente}

El personal docente que participó de los talleres son personas que tienen larga trayectoria y experiencia en la docencia cuya edad promedio ronda los 45 años y la duración promedio de su carrera docente es de 18 años. Por lo tanto, han sido testigos de grandes transformaciones en el abordaje del tema dentro del sistema educativo público. Reconocen que anteriormente las condiciones permitían que la agricultura se abordara con mayor tiempo y de mejor manera, inclusive se aprendía de manera práctica. La mayoría de las personas docentes participantes fueron estudiantes de centros educativos del cantón.

La actividad agrícola y el medio ambiente son concebidos como aspectos fundamentales de la comunidad y de la familia, existe una conciencia de protección al ambiente relacionada con el trabajo de la tierra. :

“(...) mis papás son de la zona, creo que es lo que nos dio de comer y nos ayudó muchísimo a todos a salir adelante" (Participante 12).

Se destaca la manera en que ha cambiado la forma de producción agrícola a través del tiempo. Se sembraba en los hogares, no existía la necesidad de estar comprando en los supermercados ni en las ferias, porque los alimentos estaban al alcance y el autoconsumo era la vía de subsistencia.

"Lamentablemente ya no es tan natural como hace unos años, porque al menos mami siempre me dice a mí, porqué será que ahora los jóvenes son tan enfermizos, porque todo les cae mal, porqué padecen tanto del estómago. Nosotros no, nosotros no éramos así, pero yo le digo a mami, es que ahora todo lo que nosotros consumimos, la mayoría son químicos." (Participante 2)

Los docentes expusieron que la incorporación de la agricultura en los nuevos planes de trabajo es parte de una decisión que corresponde al Ministerio de Educación Pública (MEP) y que el nuevo programa de estudios ha sustituido la enseñanza de lo que anteriormente fue la materia de Agricultura, la cual se impartía en dos lecciones semanales, 
para cada nivel de segundo ciclo. De acuerdo con la participante 5 (2016), actualmente la cantidad de tiempo que se utiliza para estudiar la materia es mínima y las formas en las que se aborda resultan ser poco efectivas. Según su opinión, las vías de aprendizaje tienen más sentido a partir de programas prácticos que teóricos.

Con respecto al grado de satisfacción sobre la manera en cómo actualmente se imparte el tema, un informante manifestó que, si tuviera que cambiar algo, sería trabajar la agricultura como una materia aparte; mientras que la preferencia de otros dos informantes se orienta hacia la práctica: "Sería mejor de manera más vivencial" (Participante 5), "Lo haría de forma práctica, directamente en una huerta" (Participante 7).En relación con dichas afirmaciones, Martínez y Eugenio (2016) enfatizan en que los procesos de aprendizaje de la agroecología en la infancia deben impulsarse a través de "enfoques vivenciales y convivenciales, integradores y significativos, con la perspectiva de abrir un debate que nos permita avanzar en la construcción de una Agroecología transformadora".

Estos autores recalcan sobre los esfuerzos que deberían hacerse por propiciar entornos naturales como escenarios educativos en edades tempranas. Asimismo, destacan las transformaciones significativas que en las últimas décadas se han dado en el entorno cercano donde se desarrollan los procesos de aprendizaje de la infancia, los cuales se resumen en familia, barrio y escuela. Lo anterior concuerda con las opiniones de varios docentes, tal como se expuso.

El tiempo que se le dedica al tema durante el año escolar no está definido. Algunos concordaron en que se aborda de manera transversal, ya que por el contexto en el que viven es un tema que sale a la conversación. Se supone que para las clases de ciencias se debe de impartir una cierta cantidad de lecciones relacionadas con la agricultura, e inclusive en el libro que utilizan generalmente, existe un pequeño apartado referente a la agricultura, pero se aborda solamente si queda tiempo, ya que no es una prioridad. Sin embargo, no existe un programa que establezca el tiempo específico que se debe dedicar a este tema.

A pesar de que cada escuela tiene sus particularidades, todos los docentes concordaron en que lo más importante es que desde cualquier materia en que se aborde el tema de la agricultura, se debe partir del contexto rural en el que viven. Es muy común que la agricultura sea tema de conversación de cualquier materia, siendo los estudiantes los que expresan el conocimiento que tienen, ya que lo han aprendido de sus padres. En este sentido, el entorno en el que crecen y se desarrollan forma parte de las anécdotas y vivencias, de las costumbres y tradiciones de los estudiantes. Estas memorias se convierten 
en una herramienta para los docentes, por ejemplo, algunos percibieron como una fortaleza el hecho de que esta práctica sea parte del entorno y del diario vivir del estudiantado:

"O sea aquí todo está como muy inmerso en que es eso, la agricultura, los animales, la crianza de animales, las vacas. Entonces ya por ende la vaca, el pasto. Niña es que si el tiempo no está bonito y hay que echar pasto y hay que ir a cortar. Entonces ya usted se tiene que meter en ese mundo porque es la idiosincrasia del pacayense, o de la gente y la mayoría de la zona norte." (Participante 2)

Actualmente, las prácticas docentes o estrategias educativas que emplean para la construcción del conocimiento se basan en las clases teóricas, en la participación del estudiantado a partir de sus experiencias y en la utilización de distintas técnicas para el trabajo individual y grupal. Sobre estas últimas, se aprovechan las exposiciones, la asignación de pequeños proyectos (aunque se realizan muy poco), las investigaciones, las tareas enviadas al hogar y esporádicamente la participación en ferias científicas.

Según una informante, el tipo de actividades para el estudio de la agricultura se han reducido. Por ejemplo, una actividad que antes se realizaba era salir a plantar un árbol, mientras que ahora se requiere de todo un procedimiento para sacar a los y las estudiantes de la escuela. A ello se suma que la infraestructura de muchos centros educativos ya no se presta para cultivar, porque no hay espacio.

Sin embargo queda en evidencia que existe una gran apertura y voluntad por parte de los docentes participantes en propiciar espacios distintos a las aulas para el proceso de "enseñanza-aprendizaje" de la Agroecología, ya que esto generaría motivación para el estudiantado. Aunado a esto, abogan porque la enseñanza sea contextualizada y que los contenidos se ajusten con los intereses de los niños y niñas, y con la realidad del entorno.

Druger (citado en Mujica, Suarez y Rodríguez, 2015) destaca que:

“...existen muchas maneras para abordar la motivación del educando, donde la proporción de herramientas, el estímulo y el uso de la experiencia son un factor clave para lograr despertar interés en el individuo que aprende, ya que se pueden olvidar los contenidos teóricos pero no las experiencias. De esta manera el estudiante, debe incrementar progresivamente sus potencialidades, donde los docentes deben estimularla adquisición de hábitos para el estudio y el trabajo que le permitan alcanzar los conocimientos de manera activa y responsable al adquirir habilidades para la autogestión del propio aprendizaje." 
Con respecto a la ejecución de un huerto escolar, algunos docentes destacaron la importancia de contemplar los factores que lo podrían limitar, tales como el tiempo, la disponibilidad y disposición por parte de los docentes y la falta de un acompañamiento por parte de una institución gubernamental o de alguna empresa externa que pueda asumir un proyecto de este tipo. Las docentes de un centro educativo enfatizaron que el elemento primordial es el compromiso por parte de las personas para ejecutar un proyecto, de lo contrario sería muy difícil lograrlo.

Refiriéndose al papel preponderante del educador para la puesta en marcha de huertos escolares, Torres y Del Valle enfatizan que "deben ponerse en marcha también programas de formación permanente para el profesorado, incluyendo incentivos que pongan en valor la iniciativa" (s.f, p.11). De esta manera, se estaría reconociendo la ardua labor que implica para una persona docente realizar proyectos de este tipo. Esto podría motivar al profesorado, ya que es importante visibilizar el tiempo que implica preparar proyectos de este tipo.

La evaluación de los aprendizajes se ha hecho algunas veces mediante proyectos específicos, o bien, a través del trabajo cotidiano. Los informantes se refirieron en concreto a la materia de ciencias, que se evalúa mediante dos pruebas teóricas trimestrales por medio de exámenes, y en uno de ellos, se incluye el tema de la agricultura. Un informante hizo la excepción de que inclusive se ha evaluado en Estudios Sociales.

Por último, la actitud y aptitud de una persona docente es determinante en la ejecución de acciones para la enseñanza de la agroecología. Por ello, resulta importante destacar a Mujica (citado en Mujica, Suarez y Rodríguez, 2015) quien expone lo siguiente:

"La mayor satisfacción para un profesor será haber ayudado a sus estudiantes a encontrar el lugar donde puedan ser útiles en la vida, pero sobre todo a que participen de manera activa en el progreso de las comunidades rurales y estén abiertos para la producción sostenible en el campo, es decir, que la más pequeña experiencia vivida puede tener un efecto monumental en su desarrollo personal y profesional dentro de la sociedad actual".

\subsection{Experiencia del diplomado Alimentación, Comunidad Aprendizaje, del programa Laboratorios para la Vida}

El diplomado de formación dirigido a docentes Alimentación, Comunidad y Aprendizaje se creó a través de un programa que hoy en día se conoce como Lab-vida (ya que en un 
inicio llevaban por nombre Laboratorios para la Vida) desde hace aproximadamente 12 años, a través de un equipo interdisciplinario conformado y dirigido por personal académico del Colegio de la Frontera Sur (ECOSUR) y del Centro de Investigaciones y Estudios Superiores en Antropología Social (CIESAS), en el municipio de San Cristóbal de las Casas, del estado de Chiapas en México (Labvida, 2017). Lab-vida está formado por profesionales pertenecientes a las ramas de la antropología, biología, salud alimentaria, pedagogía, agroecología, desarrollo rural y gestión ambiental, que además se han especializado en métodos de educación popular, con el objetivo de trabajar con docentes.

La investigadora tuvo la oportunidad de ser partícipe como voluntaria colaboradora del equipo Lab-vida en la primera semana intensiva de la promoción del diplomado del año 2016. Para esta ocasión, las personas docentes inscritas provenían en su mayoría de centros educativos de primaria del estado de Chiapas.

Este diplomado ha representado una experiencia enriquecedora y una oportunidad para conocer la manera en que se ha venido trabajando la agroecología, los sistemas agroalimentarios locales y los huertos escolares en otras regiones, con docentes de centros educativos, mediante procesos de enseñanza-aprendizaje que proponen una serie de metodologías basadas en la investigación de la realidad, las experiencias vivenciales, la experimentación, el trabajo colectivo y participativo y otro tipo de actividades prácticas que vienen a complementar los métodos tradicionales de enseñanza en la educación formal que se sustentan en la teoría.

En cuanto a la organización del diplomado, es importante indicar que para lograr un abordaje integral trabajan sobre 5 ejes temáticos que son: alimentación consciente, agroecología, conocimiento local y diálogo de saberes, investigación y actitud científica e iniciativas educativas duraderas en la comunidad. Asimismo, han adoptado cinco modelos de enseñanza y aprendizaje, que son: formador de formadores, comunidad de aprendizaje, campesino a campesino, educación popular y el método inductivo intercultural (Labvida, 2016).

Según el equipo formador del programa Labvida, no existe un único camino o modelo a seguir para la enseñanza-aprendizaje, y en el caso específico del estudio de la agroecología, resulta importante utilizar diferentes modelos que parten del conocimiento y experiencias que traen consigo las personas, y a través de los cuales se aprende en conjunto y de manera horizontal. 
Para la elaboración de la propuesta que derivó de la investigación interesaron algunas técnicas de estos modelos formativos. La investigadora adoptó y adaptó algunos ejercicios y técnicas participativas de educación popular para el diseño de la propuesta. Lo que este modelo busca es desarrollar procesos de aprendizaje que permitan la discusión y reflexión colectiva, socializar el conocimiento individual y potenciar el conocimiento colectivo (Vargas, 1999). De igual modo, utilizó el método inductivo intercultural para algunos temas.

La Dra. Helda Morales (comunicación personal, 15 de julio, 2016), quien es investigadora y una de las facilitadoras y creadoras del diplomado, considera que la implementación de los huertos escolares por sí solos no ha generado el impacto deseado. Si bien es cierto que la enseñanza a través del huerto escolar es una práctica vivencial efectiva de "aprender haciendo", que se ha vinculado a los programas de enseñanza primaria como un aula externa, las personas docentes se han encontrado con algunas dificultades en el camino a la hora de establecer huertos, como por ejemplo, darles continuidad y mantenimiento, sobre todo cuando los ciclos lectivos se interrumpen durante el año.

Actualmente, el programa Lab-vida abarca la agroecología de una manera más integradora. Además del abordaje que tradicionalmente se le ha dado desde las ciencias naturales, el estudio también se enfoca desde las ciencias sociales, ya que en primera instancia se debe comprender que un sistema agroalimentario local tiene un fuerte componente cultural. Por esta razón, una de las prioridades del diplomado consiste en impulsar y fomentar la restauración cultural de los sistemas de producción de alimentos en las escuelas chiapanecas a través del rescate y fortalecimiento de los saberes de las comunidades. Suele suceder que el conocimiento popular que existe detrás de estas prácticas tradicionales se reconoce poco, e incluso muchas veces está invisibilizado, hasta entre los mismos pobladores.

Es por ello que se parte de una pedagogía vivencial que busca colectivizar el conocimiento local y propiciar el diálogo de saberes, al mismo tiempo que se enlaza con el conocimiento "universal" o cosmopolita (occidental). Con ello, se busca retomar y fortalecer la dieta local, los alimentos producidos de forma limpia y natural, así como potenciar estilos de vida más sanos que propicien una alimentación más consciente y autosuficiente. Aunado a esto, se busca revalorizar al pequeño productor y promover el comercio justo.

Las técnicas o métodos de enseñanza-aprendizaje empleados parten del conocimiento y experiencias que traen consigo los habitantes de una comunidad. El trabajo de campo y la investigación a través de la observación, así como la participación activa son herramientas 
de gran utilidad para recolectar información en este método. Resulta muy importante que se generen vínculos con la comunidad a la cual pertenecen, ya que a través del contacto directo y la acción se extraen los mejores conocimientos.

De acuerdo con la doctora Morales:

"Lo más rico de la ciencia es ese proceso mental de descubrir un problema, llenarse de curiosidad de cómo se dan los procesos. Esa necesidad de hacer una ciencia localizada, no es lo mismo en un lugar que en otro. Como que no hay respuestas tan universales, hay que hacerlas a un nivel más local. Hacerse preguntas de cómo es el sistema alimentario donde yo trabajo, mi comunidad, mi estado, mi país...". (Comunicación personal, 15 de julio, 2016).

\section{Conclusiones}

A través de la presente investigación se logró comprobar que en la zona estudiada no existe una formación exclusiva, suficiente y pertinente orientada hacia el estudio de los sistemas agroalimentarios locales, y por ende resulta apremiante la creación de espacios de acción, reflexión y sensibilización sobre la realidad del sistema agroalimentario que impera en la actualidad en el cantón de Alvarado.

El trabajo con el estudiantado permitió determinar el nivel de conocimiento que tienen sobre el tema y la manera en que perciben el sistema productivo de sus comunidades. Los y las estudiantes mostraron una gran capacidad crítica y analítica para recibir y procesar información. Por lo tanto, están en condición de recibir una formación enfocada en fortalecer y potenciar estos conocimientos.

Además, por parte del profesorado existe un gran interés por contar con alternativas pertinentes que se puedan articular con el proceso de enseñanza-aprendizaje. Reconocen la necesidad de estudiar la agricultura desde la Educación General Básica porque consideran que es necesario fortalecer estos conocimientos. El aporte del cuerpo docente constituyó una base sobre la manera en que se puede abordar la temática en estudio, tomando en cuenta las limitaciones en cuanto al factor tiempo, el recurso humano y el material disponible.

A partir del análisis de los resultados obtenidos y del trabajo de la experiencia del diplomado, se concluye que para estudiar el tema de la agroecología se deben implementar distintas metodologías de aprendizaje a través de la acción y de la construcción colectiva del conocimiento. Esto se refuerza con el aporte del cuerpo docente de las escuelas 
participantes que han afirmado, por la experiencia que tienen, que el aprendizaje a través de la teoría por sí sola no resulta efectivo.

Tomando en cuenta lo expuesto por ambos grupos de informantes con respecto a las materias en las que se ha estudiado el tema, resultó fundamental revisar los planes de estudio de segundo ciclo de Ciencias y Estudios Sociales-Cívica. A partir de esto, se confirmó la pertinencia de actualizar el currículum educativo con el contenido de la agroecología, así como la necesidad y factibilidad de articularlo con estrategias de Educación Ambiental.

El producto elaborado resultó en una guía educativa de apoyo para el profesorado del circuito $\mathrm{N}^{\circ} 4$ escolar de la provincia de Cartago. En ella se han planteado dinámicas y ejercicios prácticos para el aprendizaje, puntuales e independientes entre sí, de manera que puedan ajustarse al tiempo disponible y a los objetivos de la materia según el plan de estudios. Esto permite que se puedan retomar y continuar en otros trimestres. La propuesta incluye la implementación de huertos escolares como una estrategia educativa más, entre otra gama de opciones para estudiar el tema. ${ }^{3}$

A la hora de implementar estas guías se debe tener en cuenta, en todo momento, que las personas de la comunidad son una fuente de información muy valiosa. La participación de las personas adultas mayores de las comunidades es indispensable para la construcción de procesos de enseñanza-aprendizaje, ya que son portadoras de costumbres y tradiciones en el tema en estudio. Asimismo, dentro de este proceso de aprendizaje resulta esencial vincular la agricultura con la cocina tradicional, el comercio justo y la salud alimentaria, los cuales representan la base de la soberanía alimentaria que se fundamenta en la filosofía del buen vivir. A su vez, la soberanía alimentaria tiene un fuerte componente ligado a la salud ambiental, ya que dentro de sus principios está la necesidad de una producción agrícola sostenible y ecológica, tendiente a garantizar entornos sanos y proveer alimentos inocuos y nutritivos.

Un aspecto implícito en el tema en estudio es la importancia de impulsar el desarrollo local tendiente a favorecer a los pequeños y medianos productores, y a fortalecer los encadenamientos productivos que pueden generar beneficios a la comunidad. Por esta

\footnotetext{
${ }^{3}$ En el Anexo $\mathrm{N}^{\circ} 1$ se exponen un par de ejemplos de guías tomados de la propuesta. En total se presentan cuatro guías para la materia de Estudios Sociales-Cívica y nueve guías para Ciencias, para un total de 13 guías didácticas para abordar el tema según el eje temático elegido. Además de esto la guía consta de una presentación, con detalles del cantón y de la eliminación de la materia de agricultura. En ella se incluye en qué consiste la propuesta, por qué se escoge Cartago circuito 4, cómo fue posible, sus objetivos y los principales conceptos en el referente teórico.
} 
razón, la propuesta generada se fundamentó en un abordaje integral e indisoluble de la agroecología, la soberanía alimentaria, la salud ambiental y el desarrollo local.

Como resultado de la investigación se concluye que la implementación de un huerto escolar por sí solo, como única propuesta para la enseñanza-aprendizaje de la agroecología, podría no tener el impacto esperado. Esta aseveración se ha logrado a través de un proceso de investigación que, por su condición cualitativa, ha permitido la construcción del conocimiento por aproximaciones, producto de la triangulación.

\section{Agradecimientos}

Quiero agradecer a la M.Sc Xinia Alvarado Zeledón y al M.Ed Wilfredo Gonzaga Martínez por asesorarme durante todo el proceso de la tesis; al personal docente, administrativo y estudiantes de las Escuelas Guillermo Rodríguez Aguilar, Encarnación Gamboa Piedra y Presbítero Juan de Dios Trejos porque sin su apoyo y aporte esta investigación no hubiera sido posible; al Equipo de Laboratorios para la Vida, a mi madre y a mi padre -mis maestros de vida- por darme una educación e impulsarme a seguir, y muy especialmente un profundo agradecimiento a la M.Sc Rocío Rodríguez Villalobos por ser una guía incondicional, por su entrega y compromiso para sacar adelante la investigación.

\section{Referencias}

Abarca, Allan, Alpízar, Felipe, Sijaba, Gina y Rojas, Carla. (2013). Técnicas cualitativas de investigación. San José, Costa Rica: Editorial de la Universidad de Costa Rica

Carvajal, Erick. (19 de marzo de 2013). La agricultura se marchita en las escuelas de Costa Rica. CR Hoy. Recuperado de http://www.crhoy.com/agronomos-denuncian-que-elmep-elimino-la-materia-de-educacion-agricola/

Colás, María Pilar, Buendía, Leonor. (1998). Investigación Educativa. Sevilla, España: Editorial Alfar

De la Cruz, Elba, Ruepert, Clemens, Wesseling, Catharina, Monge, Patricia, Chaverri, Fabio, Castillo, Luisa y Bravo, Viria (2004). Los plaguicidas de uso agropecuario en Costa Rica: impacto en la salud y el ambiente. Heredia, Costa Rica: IRET-UNA

Gaudiano, Edgar. (30 de mayo de 1999). El ambiente: mucho más que ecología. Suplemento Niños de El Universal. Recuperado de http://www.anea.org.mx/el-ambiente-muchomas-que-ecologial

Gosselin, Pierre, Furgal, Chris, Ruiz, Alfonso. (2001). Indicadores básicos de Salud Ambiental para la Región de la Frontera México- Estados Unidos. Texas, Estados Unidos: OPS. 
Gurdián-Fernández, Alicia. (2007). El Paradigma Cualitativo en la Investigación SocioEducativa. San José, Costa Rica: Coordinación Educativa y Cultural CentroamericanaAgencia Española de Cooperación Internacional.

Hernández, Roberto, Fernández, Carlos, Baptista, Pilar (2008). Metodología de la Investigación. D.F., México: Mc Graw Hill.

La Vía Campesina. (15 de enero del 2003). ¿Qué es la Soberanía Alimentaria? [Entrada en blog]. Recuperado de https://viacampesina.org/es/que-es-la-soberania-alimentaria/

Labvida (2017). Diplomado: Alimentación, comunidad y aprendizaje. Recuperado de: http://redhuertos.org/Labvida/resenas-diplomado/convocatoria-2017-2018/

Ley de Desarrollo, Promoción y Fomento de la Actividad Agropecuaria Orgánica, N 8591. (2007). Recuperado de http://www.pgrweb.go.cr/scii/Busqueda/Normativa/Normas/nrm texto completo.aspx?p aram1=NRTC\&nValor1=1\&nValor2=60741\&nValor3=0\&strTipM=TC

Martínez-Madrid Belén y Eugenio, Marcia (2016). Acercamiento a la Agroecología en la Infancia: propuestas educativas y reflexiones. Revista Agroecología, 11(1), 7-18.

Ministerio de Agricultura y Ganadería, Agencia de Servicios Agropecuarios Pacayas. (2015). Diagnóstico AEA Pacayas. Cartago, Costa Rica: MAG.

Ministerio de Educación Pública. (2004). Transversalidad en el Currículo Educativo Costarricense San José, Costa Rica: MEP.

Ministerio de Salud, Secretaría de Ambiente y Desarrollo Sostenible, Organización Panamericana de la Salud, Asociación Argentina de Médicos por el Medio Ambiente AMMA. (2007). La problemática de los agroquímicos y sus envases, su incidencia en la salud de los trabajadores, la población expuesta por el ambiente. Recuperado de https://www.fundacionfemeba.org.ar/blog/farmacologia-7/post/la-problematica-de-losagroquimicos-y-sus-envases-su-incidencia-en-la-salud-de-los-trabajadores-lapoblacion-expuesta-por-el-ambiente-1a-ed-43313

Mujica Henry, Suarez Marlem, Rodríguez Alberto (2015). Aulas Agroecológicas. Alternativa de Enseñanza para Escuelas Rurales. Saber. Revista Multidisciplinaria del Consejo de Investigación de la Universidad de Oriente, 27(1), 120-129.

Municipalidad de Alvarado, Costa Rica. (2015). Plan Regulador Territorial del Cantón de Alvarado. Recuperado de http://www.munialvarado.go.cr/index.php/mnconozcanos/mn-micanton/mn-planregulador

Naranjo, Francisco. (2010). La problemática ambiental del lavado de la papa. Éxito Empresarial, (110), 1-4.

Organización de las Naciones Unidas para la Agricultura y la Alimentación. (2010). Nueva Política de Huertos Escolares. Roma, Italia: FAO 
Fundación Vivo Sano (s.f.). Salud en la Escuela: por una vida más sana [Entrada en blog]. Recuperado de https://www.vivosano.org/salud-ambiental-la-escuela/

Organización de las Naciones Unidas para la Educación, la Ciencia y la Cultura, Programa de Naciones Unidas para el Medio Ambiente, Programa Internacional de Educación Ambiental. (1997). Actividades de Educación Ambiental para las Escuelas Primarias. Recuperado de https://unesdoc.unesco.org/ark:/48223/pf0000096345 spa

Organización Panamericana de la Salud. (2003). Glosario de Salud Ambiental en español. [Archivo PDF] Lima: OPS-OMS. Recuperado de http://www.bvsde.paho.org/bvsair/e/manuales/glosarioES.pdf

Organización Panamericana de la Salud, (2004). De la teoría a la práctica: Indicadores de Salud Ambiental Infantil. Implementación de una iniciativa lanzada en la Cumbre Mundial sobre el Desarrollo Sostenible. Recuperado de https://apps.who.int/iris/handle/10665/43053

Programa de Naciones Unidas para el Ambiente. (2004). Intoxicación por plaguicidas en niños: Información para la gestión y la acción. Suiza: PNUMA.

Restrepo, José, Angel, Diego Iván, Prager, Martin. (2000). Agroecología. Santo Domingo, República Dominicana: Centro para el Desarrollo Agropecuario y Forestal (CEDAF).

Torres, Andrea y Del Valle, Julia. (Junio de 2016). Agroecología y Educación: los huertos escolares. VI Congreso Internacional de Agroecología. Congreso llevado a cabo en la Universidad de Vigo, España.

Vargas, Laura, Bustillo, Graciela. (1999). Técnicas participativas para la educación popular. San José, Costa Rica: Alforja.

Weinberg, Jack. (2009). Guía para las ONG sobre los plaguicidas peligrosos y el SAICM. Recuperado https://ipen.org/sites/default/files/documents/ngo guide hazpest saicm-es.pdf 


\section{ANEXO 1.}

Se presenta un pequeño extracto de la propuesta para estudiar la agroecología con los estudiantes del segundo ciclo escolar del circuito $^{\circ} 4$ de la provincia de Cartago. Corresponden a un total de trece guías didácticas diseñadas que tienen como objetivo contribuir con el estudio y desarrollo de los ejes temáticos o unidades seleccionados del Plan de Estudios de Ciencias y de Estudios Sociales-Cívica, a través de situaciones de aprendizaje enfocadas en el tema de la Agricultura y la Agroecología.

Propuesta para estudiar la agroecología con los estudiantes del segundo ciclo escolar del Circuito $\mathrm{N}^{\circ} 4$ de Cartago. ${ }^{4}$

\section{GUÍA DIDÁCTICA PARA EL DESARROLLO DE LA PRIMERA UNIDAD DEL PROGRAMA DE ESTUDIOS SOCIALES Y CÍVICA}

\begin{tabular}{|l|l|}
\hline $\begin{array}{l}\text { TITULO DE LA } \\
\text { UNIDAD * }\end{array}$ & $\begin{array}{l}\text { Conozcamos la historia y cultura regional para la práctica de actitudes } \\
\text { democráticas. }\end{array}$ \\
\hline PROPÓSITO* & $\begin{array}{l}\text { Que los estudiantes comprendan la importancia de la historia de su región para } \\
\text { entender el presente, destacando algunas actividades económicas realizadas, } \\
\text { hechos y personajes. Asimismo, se busca establecer vínculos con los espacios } \\
\text { democráticos de la región, para la construcción de ideales en común que } \\
\text { permitan la vivencia de prácticas ciudadanas. }\end{array}$ \\
\hline $\begin{array}{l}\text { APRENDIZAJES } \\
\text { INDIVIDUALES Y Y } \\
\text { COLECTIVOS POR } \\
\text { LOGRAR }\end{array}$ & $\begin{array}{l}\text {-Reconocimiento de la importancia de revalorar la historia de la región } \\
\text { relacionada con la agricultura y las tradiciones alimentarias como parte de la } \\
\text { identidad comunitaria. } \\
\text {-Desarrollo de destrezas y aptitudes relacionadas con los sistemas } \\
\text { agroalimentarios locales a través de un aprendizaje vivencial. } \\
\text {-Revalorización y rescate de prácticas y manifestaciones culturales heredadas, } \\
\text { que son la base de los sistemas de producción alimentarios y de la cocina } \\
\text { tradicional de la región. } \\
\text {-Apreciar y valorar los conocimientos y prácticas tradicionales que constituyen la } \\
\text { base de un sistema agrícola basado en prácticas ecológicas. }\end{array}$ \\
\hline
\end{tabular}

${ }^{4}$ La Guía Didáctica dirigida al profesorado tiene como objetivos principales los siguientes: contribuir con el estudio y desarrollo de los ejes temáticos o unidades seleccionados del Plan de Estudios de Ciencias y de Estudios Sociales-Cívica, a través de situaciones de aprendizaje enfocadas en el tema de la Agricultura y la Agroecología; generar espacios de acción-reflexión que faciliten la comprensión del sistema agroalimentario actual, y con ello la importancia de plantear alternativas ecológicamente responsables; propiciar un abordaje integral de la agroecología desde la parte biológica, socio-económica y cultural enfatizando su relación con la Salud Ambiental; promover el uso de diferentes metodologías basadas en la investigación-acción participativa y en el diálogo de saberes, con el fin de enriquecer el proceso de construcción colectiva y horizontal del aprendizaje y crear ambientes que fomenten el trabajo en equipo, la creación de responsabilidades, la solidaridad, la conciencia ecológica y el compromiso. 


\begin{tabular}{|c|c|}
\hline $\begin{array}{l}\text { CONTENIDOS } \\
\text { CURRICULARES }\end{array}$ & $\begin{array}{l}\text { - Conceptuales: } \\
\text {-Características naturales, históricas, sociales, económicas y culturales que } \\
\text { definen la actividad agrícola de la zona norte de la provincia de Cartago } \\
\text {-Aportes del cantón al desarrollo socio-económico agrícola de la región central } \\
\text { de Costa Rica. } \\
\text {-Sistemas alimentarios tradicionales de mi región: } \\
\text {-Su relación con la agricultura basada en prácticas ecológicas, la economía local, } \\
\text { el desarrollo sostenible, la alimentación sana y la salud comunitaria. } \\
\text {-Técnicas, prácticas y saberes agrícolas heredados. } \\
\text {-Sistemas agroalimentarios locales basados en prácticas ecológicas: "La Milpa" } \\
\text {-Cocina local: Comidas tradicionales y la materia prima (cultivos), alimentos } \\
\text { subutilizados. } \\
\text { - Procedimentales: } \\
\text {-Reconocimiento de los aspectos que definen la alta productividad agrícola de la } \\
\text { zona norte de Cartago. } \\
\text {-Comprensión y diferenciación de la importancia de la agricultura de la zona en el } \\
\text { desarrollo de la región y del país. } \\
\text {-Reconocimiento de los aspectos más importantes que caracterizan el sistema } \\
\text { alimentario tradicional de la región. } \\
\text {-Comprensión de la relación entre la agricultura basada en prácticas ecológicas y } \\
\text { las prácticas agrícolas tradicionales de la región. } \\
\text {-Identificación y caracterización de la base de la cocina tradicional. } \\
\text {-Diferenciación entre el sistema agrícola tradicional de la región y el sistema } \\
\text { agrícola actual. } \\
\text { - Actitudinales: } \\
\text {-Valor del aporte económico, cultural y social de la actividad agrícola de la región } \\
\text { en el resto del país. } \\
\text {-Aprecio por las características geográficas, naturales, sociales y culturales que } \\
\text { definen la riqueza agrícola del cantón y su historia. } \\
\text {-Respeto y valor en relación con la importancia de preservar, fortalecer y/o } \\
\text { rescatar las prácticas culturales que definen el sistema alimentario tradicional de } \\
\text { la comunidad. } \\
\text { - Aprecio por las bondades de los principios de la agricultura basada en prácticas } \\
\text { ecológicas como una forma de fortalecer el sistema agroalimentario local. } \\
\text {-Conciencia e interés sobre los efectos negativos de los sistemas alimentarios } \\
\text { actuales en la salud humana y en el medio ambiente. }\end{array}$ \\
\hline $\begin{array}{l}\text { VALORES } \\
\text { ACTITUDES } \\
\text { COMPORTAMIENT } \\
\text { OS ÉTICOS, } \\
\text { ESTÉTICOS Y } \\
\text { CIUDADANOS } \\
\text { QUE SE } \\
\text { ABORDAN }\end{array}$ & $\begin{array}{l}\text { - Respeto por el espacio geográfico: } \\
\text {-Aprecio por las características de la región que definen la actividad agrícola del } \\
\text { cantón } \\
\text {-Reconocimiento de la producción agroalimentaria local como un aporte para la } \\
\text { economía nacional. } \\
\text { - Pensamiento crítico: } \\
\text {-Reflexión sobre la necesidad de retomar prácticas culturales y conocimientos } \\
\text { relacionados con los sistemas agrícolas y las cocinas tradicionales de la región. } \\
\text {-Análisis del uso adecuado de los recursos naturales en las actividades } \\
\text { productivas. } \\
\text {-Responsabilidad de instaurar un sistema agroalimentario local que sea } \\
\text { sostenible para las futuras generaciones y que garanticen el derecho a un } \\
\text { ambiente sano y ecológicamente equilibrado (a través de una agricultura } \\
\text { emergente basada en principios ecológicos) }\end{array}$ \\
\hline
\end{tabular}




\begin{tabular}{|l|l|}
\hline & $\begin{array}{l}\text {-Soberanía alimentaria: una agricultura emergente basada en el respecto por los } \\
\text { sistemas agroalimentarios locales y el desarrollo comunitario. } \\
\text { - Creatividad e innovación: } \\
\text {-Agricultura basada en prácticas ecológicas como un espacio para la } \\
\text { recuperación de saberes. } \\
\text {-El aprendizaje a través de actividades participativas vivenciales y horizontales } \\
\text { construidas de manera colectiva entre la comunidad. }\end{array}$ \\
\hline $\begin{array}{l}\text { ACTIVIDADES DE } \\
\text { APRENDIZAJE }\end{array}$ & $\begin{array}{l}\text { - El maíz, herencia mesoamericana. } \\
\text { - Investigando sobre comidas tradicionales a base de maíz. } \\
\text { - El calendario socionatural sobre el ciclo de la milpa. } \\
\text { - Tarjetas de interapredizaje. } \\
\text { - Historias y cuentos del maíz. } \\
\text { (La explicación de cada actividad sugerida se brinda en la guía) }\end{array}$ \\
\hline
\end{tabular}

Fuente: Elaboración propia a partir de los resultados de la investigación de tesis y de las bibliografías consultadas

*Tomados textualmente del Programa de Estudios Sociales y Cívica

\section{GUÍA DIDÁCTICA \\ PARA EL DESARROLLO DE LA PRIMERA UNIDAD DEL PROGRAMA DE CIENCIAS DE QUINTO GRADO}

\begin{tabular}{|c|c|}
\hline $\begin{array}{l}\text { TÍTULO DE LA } \\
\text { UNIDAD * }\end{array}$ & $\begin{array}{l}\text { Los seres vivos en entornos saludables, como resultado de la interacción de } \\
\text { aspectos biológicos, socioculturales y ambientales. }\end{array}$ \\
\hline $\begin{array}{l}\text { CRITERIOS DE } \\
\text { EVALUACIÓN }\end{array}$ & $\begin{array}{l}\text { - Reconocer las características físicas que definen a las principales familias de } \\
\text { plantas que nos proveen los alimentos básicos e indispensables para una } \\
\text { alimentación saludable. Así como la manera en que estas se clasifican. } \\
\text { - Identificar las relaciones de interdependencia entre los factores bióticos y } \\
\text { abióticos que garantizan el equilibrio de un sistema agroecológico. } \\
\text { - Compartir y conocer las experiencias personales y comunitarias que se dan en } \\
\text { el cantón de Alvarado basadas en el uso racional de la flora y la fauna. }\end{array}$ \\
\hline $\begin{array}{l}\text { CONTENIDOS } \\
\text { ESPECÍFICOS }\end{array}$ & $\begin{array}{l}\text { El objetivo es que a través de este eje temático se puedan explicar ciertos } \\
\text { principios de la agroecología que tienen que ver con la biodiversidad, es decir la } \\
\text { diversidad de vida, de tipos, de formas, de colores, de olores, de sabores que } \\
\text { nos ofrece la naturaleza a través de los alimentos, la cual nos provee alimentos } \\
\text { limpios y variados que nos hacen crecer sanos, fuertes y felices. } \\
\text { - Introducción al maravilloso mundo de las plantas que nos proveen alimentos. } \\
\text { - Entender el tema de las relaciones interespecíficas e intraespecíficas entre } \\
\text { especies de flora y fauna relacionadas con la producción de alimentos. } \\
\text { - El mundo de los microorganismos } \\
\text { - El mundo de las abejas y la importancia de cuidarlas } \\
\text { - Experiencia o proyecto productivo que se da en la comunidad }\end{array}$ \\
\hline $\begin{array}{l}\text { SITUACIONES DE } \\
\text { APRENDIZAJE } \\
\text { SUGERIDAS }\end{array}$ & $\begin{array}{l}\text { - Mi primer herbario } \\
\text { - Material audiovisual recomendado referente al maravilloso mundo de las } \\
\text { abejas. }\end{array}$ \\
\hline
\end{tabular}

Fuente: Elaboración propia a partir de los resultados de la investigación de tesis y de las bibliografías consultadas 
Revista indizada en

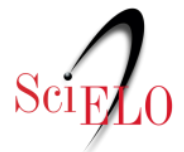

redalyc satindex

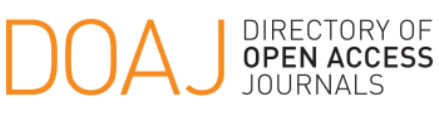

Distribuida en las bases de datos:

- Dialnet

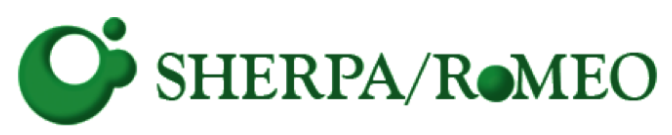

REDIB

Red Iberoamericana

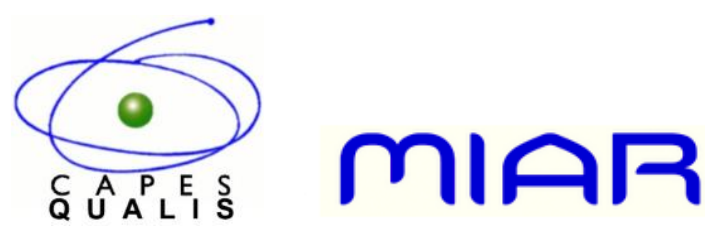

